\title{
La route géorgienne des Flandres
}

\section{Gaston Bouatchidzé}

\section{OpenEdition}

Journals

Édition électronique

URL : http://journals.openedition.org/ccs/947

DOI : $10.4000 /$ ccs. 947

ISSN : 2558-782X

\section{Éditeur :}

Presses universitaires de Rennes, Association des lecteurs de Claude Simon

\section{Édition imprimée}

Date de publication : 30 avril 2015

Pagination : 73-78

ISBN : 9782753539990

ISSN : 1774-9425

Référence électronique

Gaston Bouatchidzé, "La route géorgienne des Flandres », Cahiers Claude Simon [En ligne], 10 | 2015, mis en ligne le 22 septembre 2017, consulté le 01 mai 2019. URL : http://journals.openedition.org/ ccs/947; DOI : 10.4000/ccs.947 


\title{
LA ROUTE GÉORGIENNE DES FLANDRES
}

\author{
Gaston BOUATCHIDZÉ \\ Académie littéraire de Bretagne et des pays de la Loire
}

Avant de devenir écrivain, Claude Simon ayant esquissé une carrière de peintre, son ouvre pourrait se placer entre la plume et le pinceau: peinture, d'une part, formes graphiques des mots, de l'autre. Mais la notion de composition musicale est également souvent évoquée dans ses textes. Passer d'une langue à une autre, c'est précisément changer à la fois d'images et de sonorités. Partant, notre échange de traducteurs nous réserve des surprises. Il est d'ailleurs intéressant pour un écrivain d'interroger le reflet de sa propre œuvre dans ce miroir multicolore et polyphonique des langues du monde. C'est d'ailleurs ce que fit Claude Simon aux Deuxièmes Assises de la traduction à Arles en 1985.

Nous allons donc emprunter La Route géorgienne des Flandres tracée par la fidèle traduction d'Ilia Gasviani ${ }^{1}$. Celui-ci est rappelons-le, un traducteur passionné et un explorateur de la littérature française; le recueil Concours de jeunes traducteurs, paru en 2010 à Tbilissi, aux éditions Diogène, réunit des échantillons de traductions de différentes langues et retient, entre autres, ses traductions du français; toujours en 2010, paraît son étude: Les problèmes de composition et de versification dans "Alcools" de Guillaume Apollinaire. Mais, au fil de ce commentaire, nous ne pourrons oublier que «le traducteur propose, la langue dispose ».

Ou place son mot. L'Introduction au premier volume des Euvres de Simon ${ }^{2}$ dans la «Pléiade », signée Alastair Duncan, nous rappelle que Claude Simon ne cesse d'exploiter la polysémie des mots qui lui fournissent leur im-

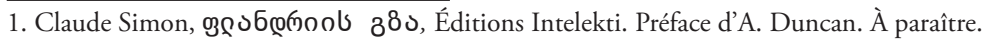

2. Claude Simon, Euvres I, éd. établie par A.B. Duncan, avec la collaboration de J. H. Duffy, Paris, Gallimard, «Bibliothèque de la Pléiade », 2006. 
pulsion. La Fiction mot à mot et d'autres discours simoniens, semés ici ou là, le confirment. La musique du texte (rythme, assonances, cadence de la phrase) accueille en vibrant une multiplicité de figures. Mais il ne faudrait pas oublier la fonction essentielle dans ces textes de la mémoire visuelle: peinture, de la toile de maître au graffiti, photographie, du portrait posé de famille aux photos de Brassaï, cartes postales, images encore et toujours prises sur le vif. Alastair Duncan observe qu'il s'agit, pour chaque texte considéré, $\mathrm{d}^{\prime}$ " une prose qui se rapproche de la poésie ». Ce travail tout à la fois pictural et poétique, Simon le définit très exactement dans La Fiction mot à mot:

Comme le peintre, et en dépit du fait qu'il n’a à sa disposition qu'une durée, l'écrivain peut cependant parvenir à « abstraire de différentes séries » des éléments qu'il assemble en une sorte de mécanique ou de système non pas bien sûr optique mais scriptural ${ }^{3}$.

Dans le Discours de Stockholm, le lauréat Nobel rappelle comment il a, en composant ses textes, prêté l'oreille à " une certaine harmonie " d'associations, d'assonances, naissant, " comme en peinture et en musique, de contrastes, d'oppositions ou de dissonances ». Et il souligne à quel point la langue elle-même est suggestive:

lorsque je me trouve devant ma page blanche, je suis confronté à deux choses: d'une part le trouble magma d'émotions, de souvenirs, d'images qui se trouve en moi, d'autre part la langue, les mots que je vais chercher pour le dire, la syntaxe par laquelle ils vont être ordonnés et au sein de laquelle ils vont en quelque sorte se cristalliser ${ }^{4}$.

En cherchant de plus à rendre compte de la composition de son "paysage intérieur ", il note que sa dimension dynamique vient de la langue:

... forcé par la configuration linéaire de la langue d'énumérer les unes après les autres les composantes de ce paysage [...], l'écrivain, dès qu'il commence à tracer un mot sur le papier, touche aussitôt à ce prodigieux ensemble, ce prodigieux réseau de rapports établis dans et par cette langue qui, comme on l'a dit, "parle déjà avant nous " au moyen de ce que l'on appelle ses " figures », autrement dit des tropes, les métonymies et les métaphores dont aucune n'est l'effet du hasard mais tout au contraire partie constitutive de la connaissance du monde et des choses peu à peu acquise par l'homme'.

La langue et les tableaux qu'elle suscite sont sans cesse sollicités. Simon se réclamant de Novalis, voit dans le langage un monde où se reflète « le jeu étrange des rapports entre les choses ${ }^{6}$ ». Et il précise: " [c']est à la recherche de ce jeu que

3. OE I, La Fiction mot à mot, p. 1194.

4. OE I, Discours de Stockholm, p. 898.

5. Ibid., p. 900.

6. Ibid., p. 902. 
l'on pourrait peut-être concevoir un engagement de l'écriture ${ }^{7} . . . »$. Il s'agit donc de progresser "laborieusement ». Même si le Discours conclut: "... nous avançons toujours sur des sables mouvants".

Posons donc le pied sur les sables mouvants de La Route des Flandres en adoptant des couleurs et des sons géorgiens. Et demandons-nous plus particulièrement comment le traducteur a résolu le passage d'une langue à l'autre, chaque fois que le texte de départ comportait une dose de cet humour si particulier de Simon qui peut voisiner avec un environnement tragique.

\section{ÉCHOS EN SÉRIES}

Quelques pages de la première partie de La Route des Flandres ${ }^{8}$ peuvent nous aider à concevoir les modalités de cet itinéraire de traduction. Georges, le narrateur, vient d'évoquer la liaison d'Iglésia et de Corinne et il revoit sa mère Sabine évoquant la famille des De Reixach:

Et par-dessus en filigrane, pour ainsi dire, cet insipide et obsédant bavardage, qui, pour Georges avait fini par être non pas quelque et d'inséparable de sa mère quoique cependant distinct [...], mais pour ainsi dire sa mère elle-même, comme si les éléments qui la composaient (la flamboyante chevelure orange, les doigts endiamantées, les robes trop voyantes qu'elle s'obstinait à porter [...]) n'avaient constitué que l'éclatant et tapageur support de ce caquetage volubile et encyclopédique, à travers lequel, au milieu d'histoires de domestiques, de couturières, de coiffeurs et d'innombrables relations et connaissances, les de Reixach, - c'est-à-dire non seulement Corinne et son mari, mais la lignée, la race, la caste, la dynastie des de Reixach- lui étaient apparus, avant même qu'il ait jamais approché l'un d'eux, nimbés d'une sorte de prestige surnaturel...» $(R F$, p. 58).

Sabine, la mère de Georges, se prête donc à un «insipide et obsédant bavardage ", laissant rouler ses trois $a$. N'en gardant que deux, le géorgien redouble la note: laklaki. Les a de bavardage et de laklaki se font écho. Esquissant une sorte de croissant de lune, le tracé de la lettre converge dans les deux alphabets. Cas unique?

Ni l'écriture simonienne, ni le tissu de la langue géorgienne ne sauraient se passer de variations répétitives. Par exemple ce bavardage qui avait « fini par être [...] [1]a mère elle-même " $(R F$, p. 58), occasion en géorgien de doubler la mise (bolos da bolos gaghda). Tapi, après n'avoir " constitué que l'éclatant et tapageur support " $(R F$, p. 58), le " que " anodin déploie ses ailes en "seulement et seulement" (mkholod da mkholod). Des mots de l'original signalant une couleur

7. Ibid.

8. La Route des Flandres, éd. Minuit, coll. « double», 2009. 
qui s'impose bénéficient soudain d'une ondoyante musicalité en traduction: «la flamboyante chevelure orange " de Sabine se métamorphose en guizguiza narindjisferi thma. "Flamboyante" se veut visible et guizguiza - audible.

Si l'on se fixe sur "les robes trop voyantes" $(R F, \mathrm{p} .58)$, on s'enlise dans une dimension visuelle intensifiée, voire un brin dramatisée, en nous disant que tvalismomtchréli kabébi correspond à la lettre à des robes à vous couper l'œil.

On se regarde dans le miroir d'une autre langue et on découvre ses propres traits invisibles. Merci à la diversité des langues (parlées et écrites) de nous offrir un précieux patrimoine global. Un coup de théâtre couronne ici l'image omniprésente de la mère. Deux dés jetés en l'honneur de la "prestigieuse " dynastie des de Reixach $(R F$, p. 59) [= Reïchak]. De quoi faire la sourde oreille au duo "des de " $s$ 'il ne réapparaissait pas, dans les lignes suivantes, sous forme de subtiles variations: "une de Reixach: de là... ». $(R F$, p. 59) De ci de là. Le "de » n’arrêtant pas de réapparaître, qu'il évoque une particule ou qu'il soit simple conjonction. Et Sabine positionnée entre sa maman et son fils Georges, la maternité se transmet de mère en mère (déda en géorgien). Donc " une de Reixach " par sa mère est bel et bien dédith erth-erthi dé reichaktagani (l'alphabet géorgien ignorant la majuscule). Variation pour piano à queue: au départ de / de, à l'arrivée dé / di / dé. Haut la tête, dès la page suivante l'original renvoie à " tel lointain ancêtre des de Reixach» $(R F, \mathrm{p} .60)$. Hélas, soumise à la configuration grammaticale de sa langue, la traduction ne garde qu'un seul $d e$. Jeu de dés.

Mais revenons aussi sur une question de phonétique qui traverse le récit. Le moment venu, la narration nous entraîne à prononcer correctement l'insolite patronyme: de Reixach. Que faire du $x$ et du ch? Simplement prononcer « $\mathrm{x}$ comme ch ", "ch comme k». C'est Iglésia, le jockey et ordonnance du capitaine qui parle:

Et lui : Reixach vingt dieux t'as pas encore compris: chac l'ixe comme ch-che et le ch à la fin comme $\mathrm{k}$ Mince alors jte jure çuilà qu'est-ce qu'il peut être cloche ça fait au moins dix fois que je lui explique t’as donc jamais été aux courses patate c'est pourtant un nom assez connu...» (RF, p. 51)

Innommables et impossibles broutilles pour l'orthographe géorgienne, à cent pour cent phonétique, c'est-à-dire transcrivant chaque son par une seule lettre. $C h$ et $k$ adoptent chacun sa lettre et le phénomène d'un $x$ réunissant en lui la sonorité d'un $k$ et d'un $s$ paraît peu figurable... Heureusement le traducteur fait preuve de courage en essayant d'expliciter l'énigme pour le lecteur. C'est là un des partis-pris possibles: expliciter sans didactisme. Quitte à coucher le vacillant patronyme d'abord en Reïksache puis de le redresser en Reïchak. Une fois adoptée, 
la bonne orthographe fait fi de toutes les hésitations, retenant désormais uniquement Reïchak. Travail sur les lettres et les phonèmes qui n'est pas facile à rendre en géorgien. Comme si on expliquait à un francophone que le mot eau (coulant de source) pourrait s'arrondir en un seul $o$, noyant dans le courant les vaguelettes en $e, a, u$.

Certes, l'inspiration aidant, il arrive à Claude Simon de frôler les images aux sonorités étrangères. À voir les mots italiens mis en exergue lorsqu'il est question d'une page de "l'épais cahier à couverture bleue " $(R F, \mathrm{p} .61)$, ayant appartenu à l'ancêtre légendaire qui figure parmi les "paperasses jaunies " montrées un jour par Sabine à son fils. Ces termes italiens ont leurs répondants français: "Candido blanc d'un blanc éclatant "; "atttegiamento geste attitude " " carnagione carnation "; "ottimo très bon "; "otremodo autrement " $(R F$, p. 62-63). Touches de couleurs et érotisme se relaient, renvoyant aux charmes féminins. Y-a-t-il alors impasse pour le traducteur? Il a beau vouloir respecter la contiguïté des termes, leur éventail visuel s'annonce plus éloquent entre deux langues sœurs (le français et l'italien) que lorsqu'il est transposé dans une langue à mille lieues des affinités romanes. Balayées, "Candide " s'efface derrière candido, " optimal " derrière ottimo et un " autre monde » derrière otremodo. Pour quoi opter? Retenir les deux versions.

\section{VARIATIONS DE COULEUR}

La palette picturale varie. L'énigmatique «bleu roi " d'un " habit de chasse " $(R F$, p. 63) devient un bleu éclatant (kachkacha lourdji feris). Le "brun-rouge " de la toile " mis à nu par une longue craquelure" $(R F, \mathrm{p} .65)$ s'adoucit en brunrougeâtre (motsitalo-kavisferi)...

Chaque fois, le traducteur a cherché à élire une expression qui, même si elle apporte une nuance par rapport au terme original, est fidèle au même type de connotation et peut s'employer dans un contexte analogue.

\section{CLICHÉS DE LANGUE}

Le traducteur est aussi confronté à la complexe question des clichés de langue, très présents dans les fragments où le texte de Claude Simon s'oralise. Comment trouver des équivalents? On le peut sans doute, mais non sans modifications connotatives: l'action souvent bascule d'une langue à l'autre. Un Français « fait d'une pierre deux coups » (RF, p. 207). Devenu Géorgien, il se doit d'abattre d'un 
coup de feu deux lièvres à la fois (erthi gasrolit or kurdguels moklavda). Sans hésiter, un Français jure "Merde pour la bibliothèque de Leipzig " (RF, p. 253) (avec une majuscule). Le Géorgien se contente de faire pleurer la mère de l'autre (amis déda vatiré). Le banal "Zut alors»(p. 205) prend de l'ampleur: que le diable le maudisse (echmakma dalakhvros). Pulsion méridionale? Allez savoir. Pareil pour "l'innommable réalité » $(R F$, p. 207) qui nous fait peur et qui devient affreuse réalité (sazaréli sinamdvilé).

Parfois il suffit de garder les mots français, surtout s'il s'agit de "grotesques silhouettes" (RF, p. 206) (groteskouli silouétébi) s'avançant sur la route de Flandres.

Il arrive aussi au traducteur de modifier légèrement une image pour la rendre plus crédible. Georges pense: "Oui, il n'y a qu'un cheval qui a pu écrire ça » $(R F$, p. 63). Pour le traducteur, le gribouilleur serait plutôt un cheval-homme (tskhenkatsa).

La polyphonie géorgienne s'accorde au texte de La Route des Flandres. La musique monte à la tête d'un buveur: " les boyaux remplis de bonne bière allemande qui fermente [...] et fait entendre un menuet de Mozart» (RF, p. 211). Que devient "fermente " en géorgien? boukboukebs.

Belle note finale de cette randonnée donquichottesque ( $R F$, p. 27) acheminant La Route des Flandres vers les sons et les couleurs des quatre coins du monde. On peut donc avancer l'idée que la traduction géorgienne adopte scrupuleusement le message de l'original, et cherche, ce qui n'est pas simple, à se plier à son esprit ou son humour, en lui offrant naturellement la tenue et l'allure de la langue d'adoption. 